\section{Kommunikation und Religion}

Kritische Anfragen an kommunikationsdidaktische Konzepte der Religionspädagogik auf Basis des kommunikationstheoretischen Ansatzes der Schule von Palo Alto. Mit einem Blick auf denkbare fundamentaltheologische Konsequenzen und auf die Unterrichtspraxis.

Wissenschaftliche Hausarbeit zur ersten Staatsprüfung für das Lehramt an Realschulen, Pädagogische Hochschule Ludwigsburg, 2002.

\section{Colin Cramer}

\begin{abstract}
Die Arbeit ist der nach vorne gerichtete Versuch, die gegenwärtig eher zurückhaltende Beschäftigung der Theologie mit dem Kommunikationsbegriff anderer Wissenschaften zu überwinden. Es ist ihre fundamentale These, dass eben dieser interdisziplinäre Diskurs Kommunikation und Religion in einen untrennbaren $\mathrm{Zu}$ sammenhang bringt. Kommunikation erweist sich als die Voraussetzung für religiöses Erleben und Handeln. D as exemplarische Darstellen einiger Schnittstellen von Kommunikation und Religion zeigt Perspektiven auf, die weitreichende Folgen für die Theologie und die Religionspädagogik implizieren.
\end{abstract}

\section{Einblick in die Arbeit}

Als wissenschaftliche Basis für die Untersuchung werden ausgewählte kommunikationsdidaktische Ansätze der Religionspädagogik (Hubertus Halbfas, Peter Biehl, Eckart G ottwald) herangezogen. Diese werden aus Sicht der kommunikationstheoretischen Schule von Palo Alto (Gregory Bateson, später auch Paul Watzlawick) betrachtet. Die Erkenntnisse aus der Projektion von abstrakter Kommunikationstheorie auf die anwendungsorientierte Kommunikationsdidaktik der Religionspädagogik ermöglicht weiterhin das Aufstellen denkbarer Konsequenzen für fundamentaltheologische Fragestellungen.

Die Untersuchung folgt demnach einem Dreischritt:

(1) Darstellung ausgewählter kommunikationsdidaktischer Ansätze der Religionspädagogik.

(2) Darstellung der Kommunikationstheorie der Schule von Palo Alto und Entwicklung deren religiösen G ehalts durch Anfragen, Ergänzungen und Revisionen zur Religionspädagogik.

(3) Entwicklung fundamentaltheologischer Konsequenzen aus dem vorigen Diskurs über Kommunikation und Religion.

Der Schwerpunkt der Arbeit liegt darin, den zu- nächst schwer erkennbaren religiösen $\mathrm{G}$ ehalt der Kommunikationstheorie Gregory Batesons freizulegen. Dabei werden wie mit einem Scheinwerfer ausgewählte Bestandteile von Kommunikation kurz beleuchtet und deren Bezug zur Religion vor dem Hintergrund der religionspädagogischen Kommunikationsdidaktik hergestellt. Religion wird nicht systematisiert, sondern vielmehr auf Grund der Systematik einer Kommunikationstheorie als Phänomen der Wahrnehmung beschrieben.

\section{Ergebnisse}

Zusammenfassend ergibt die Auseinandersetzung mit den kommunikationsdidaktischen Ansätzen der Religionspädagogik eine Fülle an medienpädagogisch relevanten Einsichten. D er Mensch findet Zugang zur Wirklichkeit über die Welt der Symbole, die neuerdings stark von den Medien geprägt werden. Subjekt und Wirklichkeit bedingen sich wechselseitig, verändem sich im kommunikativen Prozess. In diesem Zusammenhang kann auch Religion als deutender Prozess von Wirklichkeit verstanden werden. Kommunikation im Sinne von Wahrnehmung ist dabei Voraussetzung für das Ausbilden eines subjektiven Wirklichkeitsverständnisses. Religionspädagogik ist hier auch eine die Sensibilität der Heranwachsenden steigernde $\mathrm{Me}$ dienpädagogik. Dieses Wirklichkeitsverständnis bildet der Mensch auf der Schnittstelle von Lebenswelt und Transzendenz aus: Er ist religiös. Grundlage jener individuellen Konstruktion von Wirklichkeit ist das Medium der Sprache. Medienpädagogik wird demnach ähnlich der Religionspädagogik stets auch eine "Sprachschule" sein müssen. Die religionspädagogische Symboldidaktik in der Funktion einer Förderung der kritischen Wahrnehmungsfähigkeit eröffnet der Medienpädagogik neue Wege: Beide Teildisziplinen haben zur Aufgabe, Sinnmuster zur Identitätsfindung für Heranwachsende anzubahnen.

Der religiöse Gehalt der kommunikationstheoretischen Schule von Palo Alto zeigt wie folgt eine Perspektive für medienpädagogische Fragestellungen auf. D as von Bateson erstmals vermutete Prinzip der Rückkopplung betrifft auch die massenmediale Kommunikation: D ie Veränderung der Individuen durch massenmediale Nutzung wird auf das Mediensystem selbst rückgekoppelt. Dabei ist der Mediennutzer Empfänger und Sender, ist so Teil eines Kommunikationsprozesses ohne klaren Beginn und Ende.

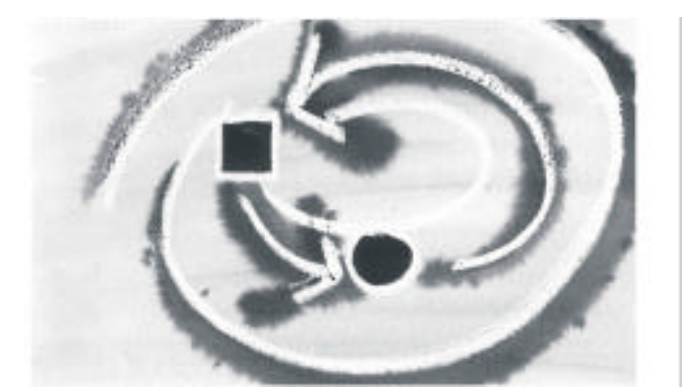


Stimmt Batesons Subjekthypothese, jeder Mensch sei ein informations- und symbolverarbeitendes System, so findet hierin jede religions- und medienpädagogische Bemühung ihre Legitimation: Heranwachsende werden als sich verändernde Individuen ernst genommen, die durch äußere Gegebenheiten beeinflusst werden können. Medienpädagogik beschäftigt sich mit Symbolen aus der Lebenswelt - deren Bedeutung hingegen reicht in den transzendentalen Bereich hinein. Dies wird deutlich, wenn Bateson Religion zwischen dem mechanischen Ursache-Wirkungs-Prinzip und einem entgegen gesetzten Glauben an Übernatürlichkeit vermutet. Die durch die Medien getragene Flucht vor der Realität hin zur Fiktion ist die Flucht von der Mechanik zur Übernatürlichkeit. Auf diesem Weg kommt der Mensch nicht an religiösen Fragestellungen vorbei. So muss sich die Medienpädagogik neben der Kommunikationstheorie zunehmend an religionspädagogischer Symboldidaktik orientieren.

Im letzten Teil der Arbeit ergeben sich die fundamentaltheologischen Konsequenzen aus einem integrativen Modell der religiösen Kommunikation, in welchem Kommunikation als der Religion vorzeitig entwickelt wird. Im Kontext des kommunikativ-religiösen Rahmens bewegen sich die Größen Mensch und Gott aufeinander zu und bilden in ihrer Schnittmenge eine Beziehung aus. Diese Wechselwirkung zeigt die untrennbare Verbindung zwischen Lebenswelt und Transzendenz, den niemals endenden Kommunikationsprozess zwischen Mensch und Gott. Unter dieser Annahme ergeben sich u.a. Konsequenzen für das Gebet und den Umgang mit der Bibel.

Die Reduzierung des gesamten Gehalts der Arbeit auf einen kurzen Satz ist wie folgt denkbar: Religion ist eine kommunikative Beziehung zwischen dem Menschen und dem Kriterium Gott, dahingehend, dass sich beide $\mathrm{G}$ rößen gegenseitig beeinflussen und sich eine Schnittmenge zwischen Kommunikation und Religion ergibt. Diese Aussage ist keineswegs trivial. Der Begriff Kommunikation vermag es, wenn auch nur in Ansätzen, erstmals eine Erklärung dafür zu geben, warum Religion als die fundamentale Kategorie des Seins bezeichnet werden kann. Religion wird zurückgeführt auf Wahrnehmung, Wirklichkeit und Existenz. O hne Kommunikation gibt es kein Bewusstsein. Mit Kommunikation aber erwacht der Mensch zum Leben, an dessen ursprünglichster Stelle er die Frage nach Sinn mit Religiosität beantwortet.

Eine solches Denkmodell und die darin verwendeten Symbolwelten scheinen eine objektive Wirklichkeit nicht erfassen zu können. Sie selbst unterliegen der individuellen Konstruktion von Realität. Aber sie öffnen den G eist für das Heilige und sind dem Menschen Hilfestellung über das zu sprechen, worüber man eigentlich nicht reden kann. 